\title{
Drum-mate: A Human-Humanoid Drumming Experience
}

\author{
Hatice Kose-Bagci, Kerstin Dautenhahn, Dag Sverre Syrdal, and Chrystopher L. Nehaniv
}

\begin{abstract}
We present an exploratory study investigating a drumming experience with Kaspar, a humanoid child-sized robot, and a human. In this work, our aim is not to have Kaspar just replicate the human partner's drumming, but to engage with the human in a 'social manner' using head gestures in a call and response turn-taking interaction and to assess the impact of nonverbal gestures on the interaction. Results from the first implementation of a human-robot interaction experiment are presented and analysed qualitatively (in terms of participants' subjective experiences) and quantitatively (concerning the drumming performance of the human-robot pair). The interaction experience is discussed in terms of imitation, turntaking, and the effect of gender differences.
\end{abstract}

Index Terms-Humanoid, robot drumming, human-robot interaction, imitation

\section{INTRODUCTION}

$\mathrm{M}$ USIC performance is a good tool for studying the interaction between humans and robots in terms of social aspects including imitation, turn-taking and synchronization. Drumming is one of the best ways of performing music in robotics, since it is relatively straightforward to implement and test, and can be implemented technically without special actuators like fingers or special skills or abilities specific to drumming.

There are several works concerning music performance in human-robot interaction. In $[1,2,3]$ robotic percussionists, play drums in collaboration with human partners. These artifacts are robot arms connected to upper torsos that are specially designed to play drums. In [4], an approach based on the movement generation using dynamical systems was tested on a Hoap-2 humanoid robot using drumming as a test case. Similarly, in [5] humanoid drumming is used as a test bed for exploring synchronization.

However, a robot will also need to motivate and sustain drumming behaviour coping with a wide range of users. One way of motivating such behaviour is through the use of social gestures. In the related field of virtual agents, researchers have shown the beneficial effects of gestures and expressions used by virtual agents both in short-term and long-term interactions

H. Kose-Bagci, K. Dautenhahn, D. S. Syrdal, and C. L. Nehaniv are with the Adaptive Systems Research Group, University of Hertfordshire, School of Computer Science, Hatfield, Herts, AL 10 9AB, U.K.

(e-mail: \{h.kose-bagci,k.dautenhahn,d.s.syrdal, c.l.nehaniv\}@herts.ac.uk).
$[6,7]$, in maintaining user involvement with the tasks encouraged by the agent.

Applied to the field of robotics, the need for the possession of a set of social skills for a robot in order to encourage behaviour successfully may require that it possesses the ability to use social cues and gestures to motivate users to interact with it. This is especially the case for assistive robotics [8].

We can already find robotic systems that use social gestures in order to encourage human-robot interaction. A well known example is KISMET where facial expressions were used to regulate the interaction with people inspired by interactions of infants with their caretakers [9]. Other recent examples include small cartoon like robotic "creatures" such as Keepon and Roillo designed to be used in interaction with children $[10,11]$. These little rubber robots have a limited action repertoire, but can produce selected gestures to engage in interaction with children in the playground. The fixed gestures are either random or tele-operated by a hidden puppeteer as in the Wizard of $\mathrm{Oz}(\mathrm{WoZ})$ technique, as a part of social interaction. Other related work is discussed in section 2.

In this study, our humanoid robot Kaspar plays drums autonomously with a human 'partner' (interactant), trying to imitate the rhythms produced by the human. However, the social interaction is not limited to the replication of drumming, but also involves studying the impact of non-verbal robot gestures which are meant to motivate the human. Kaspar produces fixed head gestures and eye-blinking as it drums. Our approach is tested with adult participants in several drumming sessions, and the experimental results are reported and analyzed below in terms of imitation, turn-taking, and the impact of gender differences.

The rest of this paper is organized as follows; in the next section, related research is summarized. Next, the methodology is briefly described. The section 4 presents the research questions, corresponding achievements and conditions. The experiments are described in the section 5 . Section 6 includes a brief conclusion on what was learned from this work, and the final section presents ideas for future work.

\section{RELATED RESEARCH}

HAILE [1,2] is a robot arm that aims to play a drum in collaboration with a human partner to study social, mathematical, physical, and technological aspects of music. HAILE does not use fixed deterministic rules, but uses 
autonomous methods to create variant rhythms. It perceives a variety of complex features of the human partner's drumming whereby a microphone on the drum analyses the sounds and produces rhythms in response.

In [3], a somewhat less musically sophisticated humanoid robot called NICO with an upper half body torso, plays a drum together with human drummers. It has visual and audio sensing to discover the right tempo, and it trains itself. It uses a simple threshold mechanism to understand the human partner's beats, and can distinguish its own performance with audio sensing, integrating the two sources of information to predict when to perform the next beat.

ROILLO is a simple robot with a rubber coated foam head, body, and an antenna. It has 3 wires connected to simple servos which move the head and body in various directions. It is used in experiments with children to study interactions between robot and children [10].

KEEPON is another simple robot, which has only a rubber head and a body. It is has a small CCD camera, and microphone on it. It can move its head, turn its body, and make bobbling actions to show its "feelings". It has both attentive and emotive actions. It is simple but robust enough to be used in play rooms in interaction with children $[11,12]$.

\section{Methodology}

In the current study the human partner plays a rhythm which Kaspar tries to replicate, in a simple form of imitation (mirroring). Kaspar has two modes: listening and playing. In the listening mode, it records and analyses the played rhythm, and in the playing mode, it plays the rhythm back, by hitting the drum positioned in its lap. Then the human partner plays again. This (deterministic) turn-taking will continue for the fixed duration of the game. Kaspar does not imitate the strength of the beats but only the number of beats and duration between beats, due to its limited motor skills. It tailors the beats beyond its skills with the minimum values allowed by its joints. Kaspar needs at least 0.3 seconds between each beat to get its joints 'ready', so that even if the human plays faster, Kaspar's imitations will be slower using durations of at least 0.3 seconds between beats. It also needs to wait for a few seconds before playing any rhythm in order to get its joints into correct reference positions.

In Fig. 1, the basic model of Kaspar-human interaction is presented. The model requires the gestures of both human and the humanoid for social interaction, as well as drumming. Currently human gestures are not detected and therefore excluded from the current implementation.

One of the fundamental problems in this scenario is the timing of the interaction; timing plays a fundamental role in the regulation of interaction (cf. [13]). It is not always clear when the robot or human partner should start interaction in taking a turn. Currently, in this model some predefined fixed time duration heuristics are used for synchronization. Kaspar starts playing if the human partner is silent for a few seconds, and tries to motivate the human partner with simple gestures.

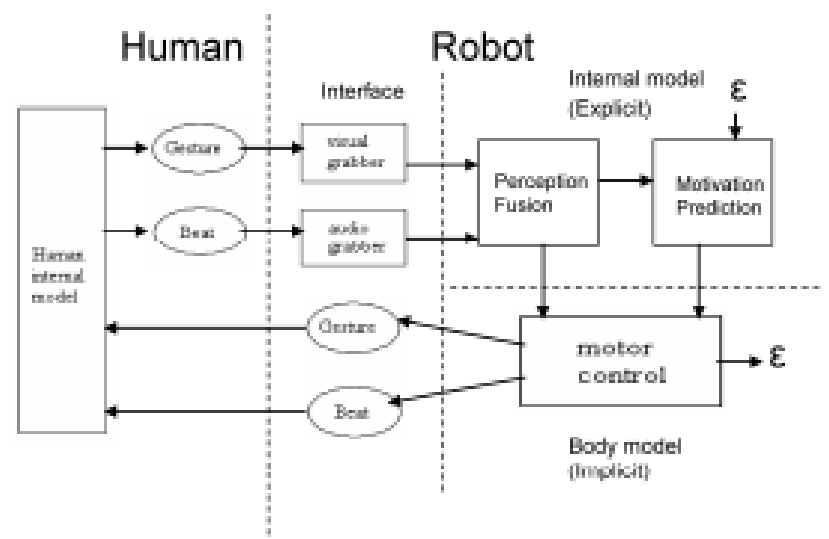

Fig. 1 The model for Kaspar-human interaction

\section{RESEARCH QUESTIONS, CORRESPONDING ACHIEVEMENTS AND CONDITIONS}

In this work, the effect of the robot's social gestures in a game of imitation, and turn-taking, was studied. A simple drumming game enriched with the robot's gestures was used as a test bed, and the subjective evaluations of the participants were analysed. Our primary focus was the possible impact that utilizing social gestures would have, not only on the game itself (in terms of performance), but also on the participant's subsequent evaluation of the game.

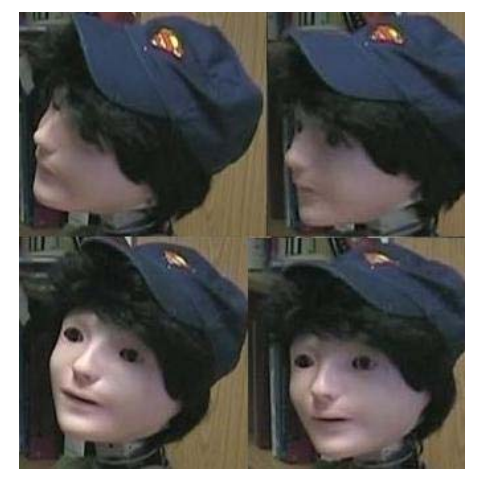

Fig. 2 Snapshots of Kaspar's dynamic head gestures used in the experimental tests

We studied three conditions with increasing amounts of gesturing. In the first condition Kaspar does not use any gestures. Kaspar only imitates the drumming. This condition was called no-gesture. In the second condition, simple head gestures (e.g. moving the head to the right or left, moving the head up or down, tilting head slightly to different angles) and eye blinking were included in Kaspar's movements (Fig. 2). Kaspar starts drumming with one of the fixed gestures. If the human partners do not play their turn, then Kaspar does not do anything, too, and the turn passes to the partner. A fixed order of $n$ gestures was used, and this order was repeated for every $n$ turns. The value for $n$ should be large enough so that the human partner does not realize that this is a fixed pattern but rather that the gestures are either meaningful or random (In 
the current experiments $\mathrm{n}$ was 7 ). This condition is called gesture in our experiments. In the third condition, Kaspar simply repeats the sequence of gestures without playing even if the partners did not play their turn. This case is named gesture+. The gestures and their sequences were the same in the last two conditions, and the drumming part was the same in all of the three conditions.

\section{EXPERIMENTS}

\section{A. Kaspar}

The experiments were carried out with the humanoid robot called Kaspar. Kaspar is a child-like humanoid robot which was designed and built by the members of the Adaptive System Research Group at the University of Hertfordshire to study human-robot interactions with a minimal set of expressive robot features. Kaspar has 8 degrees of freedom in the head and neck and 6 in the arms and hands. The face is a silicon-rubber mask, which is supported on an aluminum frame. It has 2 DOF eyes fitted with video cameras, and a mouth capable of opening and smiling, see description in [14].

\section{B. Experimental Setup}

The experiments were carried out in a separate room isolated from other people and noises which could affect the drumming experiment. Kaspar was seated on a table with the drum on its lap. The human partner was seated in front of the robot using another drum that was fixed on the table (Fig. 3). The human participants used a pencil to hit the drum. Although we suggested to the participants to use one pencil and hit on the top of the drum, sometimes they used two pencils with a single hand or with both hands, and several times they used the tambourine-style bells around the drum's sides.

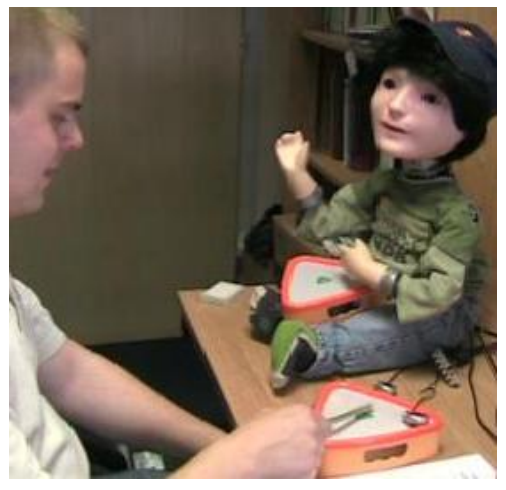

Fig. 3 A screen shot from the experiments

\section{Software Features}

The implementation of robot perception and motor control used the YARP environment [15]. YARP is an open-source framework used in the project RobotCub that supports distributed computation that emphasizes robot control and efficiency. It enables the development of software for robots, without considering a specific hardware or software environment. Portaudio [16] software was used to grab audio from the audio device, within the YARP framework.
The acoustic sound waves recorded by the sound grabber module are converted to digital music samples, which allows to use mathematical computations and sample based techniques. To detect the patterns of a sound wave, a filter based method is used, based on the work of Kose and Akin (2001) originally used to detect visual patterns.

\section{Participants}

Six female participants in the age range of 21-66, and six male participants in the age range of 24-30 took part in the study. All participants were right-handed and worked in computer science or similar disciplines at the University. They had not interacted with Kaspar prior to the experiment, and they were overall not familiar with robots. None of our participants had children, expect for one participant who had grown up children and grandchildren.

\section{E. Interaction Game Setup}

We used a one minute demo of the robot without any game where participants were shown how to interact with Kaspar. This was followed by three games reflecting the three experimental conditions described above each lasting three minutes, without indicating to the participants anything about the differences between the conditions. We used all six possible different presentation orders of the games, to analyze the effect of the order of the games on the humans. To account for possible fatigue or learning by the participants, in the sequential order section, we analyse the games according to their order number in the sequence experienced by the participants (independent of the particular experimental condition), as being the first game, second or third, disregarding their game types, e.g. for one participant the first game (number 1) would be the no-gesture game, and for another participant, it would be the third game (number 3 ).

\section{F. Evaluation of Questionnaire Data}

After the experiment the participants were asked to complete a questionnaire investigating their preferences and opinions on the three experimental conditions.

\section{1) Most and least preferred game types:}

The frequencies of participants which rated each game as most preferred can be seen below in Table 1 .

TABLE 1

MOST PREFERRED GAME

\begin{tabular}{ll}
\hline \hline Game type & Participants \\
\hline no-gesture & 2 \\
gesture & 6 \\
gesture+ & 3 \\
No preference & 1 \\
\hline \hline
\end{tabular}

Table 1 shows that the most popular game type was the gesture game, while no-gesture and gesture+ type were less preferred.

The frequencies of participants which rated each game as least preferred can be seen in Table 2.

Table 2 shows that no participants considered the gesture game as the least preferred, while the no-gesture and gesture+ 
game, had a similar number of participants which considered them the least preferred.

TABLE 2

LEAST PREFERRED GAME

\begin{tabular}{ll} 
& LEAST PREFERRED GAME \\
\hline \hline Game type & Participants \\
\hline no-gesture & 6 \\
gesture & 0 \\
gesture + & 5 \\
No preference & 1 \\
\hline \hline
\end{tabular}

\section{2) Gender Differences in most and least preferred game types:}

Most and least preferred game type according to gender are described below in Table 3 and Table 4 and in figures 4 and 5.

TABLE 3

MOST PREFERRED GAME ACCORDING TO GENDER

\begin{tabular}{|c|c|c|c|c|c|}
\hline & & & \multicolumn{2}{|c|}{ Gender } & \multirow[b]{2}{*}{ Total } \\
\hline & & & Male & Female & \\
\hline \multirow{9}{*}{$\begin{array}{l}\text { Game Type } \\
\text { Most Preferred }\end{array}$} & nogesture & Count & 2 & 0 & 2 \\
\hline & & Expected Count & .9 & 1.1 & 2.0 \\
\hline & & Std. Residual & 1.1 & -1.0 & \\
\hline & gesture & Count & 2 & 4 & 6 \\
\hline & & Expected Count & 2.7 & 3.3 & 6.0 \\
\hline & & Std. Residual & -.4 & .4 & \\
\hline & gesture+ & Count & 1 & 2 & 3 \\
\hline & & Expected Count & 1.4 & 1.6 & 3.0 \\
\hline & & Std. Residual & -.3 & .3 & \\
\hline \multirow[t]{3}{*}{ Total } & & Count & 5 & 6 & 11 \\
\hline & & Expected Count & 5.0 & 6.0 & 11.0 \\
\hline & & Std. Residual & & & \\
\hline
\end{tabular}

TABLE 4

LEAST PREFERRED GAME ACCORDING TO GENDER

\begin{tabular}{llrrrr}
\hline \hline & & \multicolumn{3}{c}{ Gender } & \\
\cline { 3 - 4 } & & & \multicolumn{2}{c}{ Male } & \multicolumn{2}{c}{ Female } & \multicolumn{2}{c}{ Total } \\
\hline Game Type Least & nogesture & Count & 1 & 5 & 6 \\
& & Expected Count & 2.7 & 3.3 & 6.0 \\
& & Std. Residual & -1.0 & 1.0 & \\
\cline { 2 - 5 } & gesture+ & Count & 4 & 1 & 5 \\
& & Expected Count & 2.3 & 2.7 & 5.0 \\
& Std. Residual & 1.1 & -1.0 & \\
\hline Total & Count & 5 & 6 & 11 \\
& & Expected Count & 5.0 & 6.0 & 11.0 \\
& Std. Residual & & & \\
\hline \hline
\end{tabular}

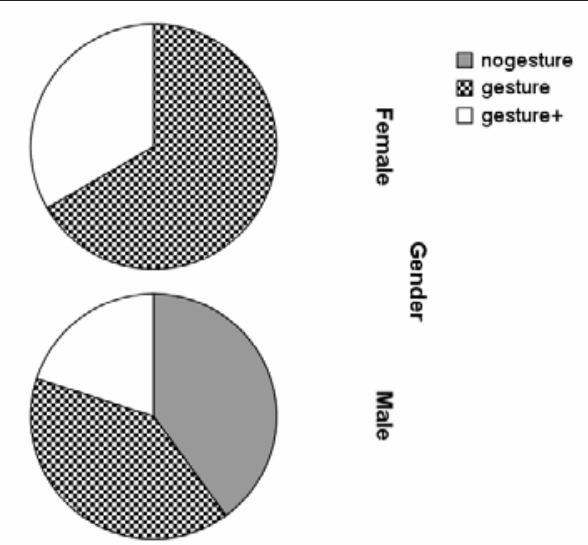

Fig. 4 Most preferred game type according to gender

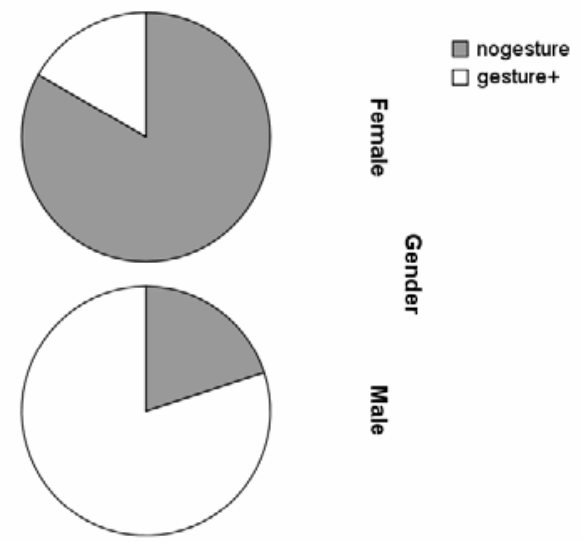

Fig. 5 Least preferred game type according to gender

The differences between males and females in most preferred game type seem to indicate that for males the preferred game type is spread evenly across the three game types, while for females the no-gesture game is not preferred by any. The differences between males and females were assessed using a likelihood ratio chi-square test. This test found no significant differences due to gender for this sample size $\left(\left(\chi^{2}(1,11)=3.70, p=.16\right)\right.$.

For the least preferred game type, however, there were significant differences due to gender $\left(\left(\chi^{2}(1,11)=4.75, \mathrm{p}=.03\right)\right.$. As table 4 and figure 5 suggests, this difference manifests as males predominantly choosing the gesture+ game type as their least preferred game type, while females predominantly chose the no-gesture game type as their least preferred game.

\section{3) Reasoning behind preferences}

While an exhaustive description of the qualitative analysis of the participants' responses is beyond the scope of this brief paper, a short description will be given below:

Two main themes emerged from the analysis, reflecting two different ways of evaluating the games. The first theme was that of task-based evaluation, in which participants would explain their choice by referring to the success of Kaspar in imitating their drumming. The second theme was that of interaction-based evaluation, wherein participants would explain their choice as to which games they preferred the most and least by referring to their enjoyment of the interaction and their general liking for the robot.

When the results from the qualitative analysis were compared to the preferences of the participants, it was clear that the task-based evaluation led participants to rate the gesture+ evaluation as their least favorite game type. Participants using an interaction-based evaluation would choose the no-gesture game type as their least favorite.

In terms of gender differences, more female participants used an interaction-based evaluation when explaining their preferences compared to male participants. There were, however, some males who used the interaction-based evaluation. As such, differences between the males and females in this sample may reflect a greater tendency in males 
to use a task-based evaluation when evaluating the game types.

\section{G. Behavioural Data}

\section{1) Sequential order}

The error is the average difference between the human's number of beats and Kaspar's number of beats in each turn. It was observed that the average error in the number of beats decreased inversely to the sequence number in order of the games presented. The participants usually tried very long and fast patterns, or they did not beat loud enough to be detected reliably (Kaspar uses a high level noise filter to filter out high inner noise coming from its joints, so it can only sense loud beats) when they started to play. Interestingly, without any external encouragement, as they got used to the game, they progressively were able to synchronize themselves to the robot better. The number of errors decreased significantly between the first and third trials $(Z=2.275, p<.05)$. Details of the results are presented in Table 5.

TABLE 5

OBSERVED BEHAVIOUR ACCORDING TO ORDER

\begin{tabular}{lrrrrrr}
\hline \hline Order & Avg. error & $\begin{array}{c}\text { Max } \\
\text { beats }\end{array}$ & $\begin{array}{c}\text { Avg. } \# \text { of } \\
\text { beats }\end{array}$ & $\begin{array}{c}\text { Avg. } \# \\
\text { turns }\end{array}$ & \\
\hline 1 & $4.1 \pm 3.6$ & 41 & $7.5 \pm 5.3$ & $17.2 \pm 6.2$ & \\
2 & $3.1 \pm 3.4$ & 37 & $6.2 \pm 4.3$ & $18.3 \pm 6.7$ & \\
3 & $2.3 \pm 1.8$ & 16 & & $4.6 \pm 2.2$ & $20.5 \pm 3.8$ &
\end{tabular}

\section{2) Interaction game type}

The gesture game had the highest average error, followed by the gesture+ game. The non-gesture game had the smallest error rate. However, the differences between games were not significant in this sample size $(\mathrm{Z}=1.01, \mathrm{p}=.27)$.

TABLE 6

OBSERVED BEHAVIOUR ACCORDING TO GAME TYPE

\begin{tabular}{|c|c|c|c|c|}
\hline Game type & $\begin{array}{l}\text { Avg. } \\
\text { error }\end{array}$ & $\begin{array}{lll}\text { Max } \\
\text { beats }\end{array}$ & $\begin{array}{l}\text { Avg. \# of } \\
\text { beats }\end{array}$ & Avg. \# of turns \\
\hline no-gesture & $2.6 \pm 2.5$ & 41 & $6.2 \pm 4.6$ & $18.6 \pm 5.8$ \\
\hline gesture & $3.5 \pm 3.8$ & 37 & $6.2 \pm 5.0$ & $18.8 \pm 5.7$ \\
\hline gesture+ & $3.3 \pm 3$ & 31 & $6.0 \pm 4.7$ & $18.9 \pm 6.4$ \\
\hline
\end{tabular}

The maximum number of beats decreased with the increasing amount of gestures in the game. The average number of beats also slightly decreased with the increasing amount of gestures in the game. The average number of turns was almost the same in all three games. The total number of beats tend to decrease as the amount of gestures in the game increased. Again, the differences between game type were not significant in this sample size. Details are presented in Table 6.

\section{3) Gender}

While the sample size makes it difficult to make any strong inferences as to differences between groups, it may be reasonable on the basis of our analysis to present the results from each gender separately. Our qualitative analysis suggests that there are differences in the dynamics when interacting with the robot and as such presenting the results from the male and female sample separately, rather than just focusing on the differences, may be more informative to the reader. However, the small number of participants makes inferential statistics problematic, and as such the following analysis is only descriptive.

\section{Sequential order}

In terms of order, in later games, the participants tended to have more turns with fewer beats, which helped them synchronize with Kaspar better - decreasing Kaspar's error rate in drumming and increasing the success of the interaction (Table 7 and Table 8).

TABLE 7

OBSERVED BEHAVIOUR OF MALES ACCORDING TO ORDER

\begin{tabular}{lcrrrrr} 
Order & Avg. error & $\begin{array}{c}\text { Max } \\
\text { beats }\end{array}$ & \multicolumn{1}{c}{$\begin{array}{c}\text { Avg. } \# \text { of } \\
\text { beats }\end{array}$} & $\begin{array}{c}\text { Avg. } \# \text { of } \\
\text { turns }\end{array}$ \\
\hline 1 & $4.1 \pm 3.3$ & 41 & & $7.8 \pm 6.0$ & $15.0 \pm 6.5$ \\
2 & $3.6 \pm 3.9$ & 37 & & $7.3 \pm 4.5$ & $15.0 \pm 5.0$ \\
3 & $2 \pm 0.7$ & 11 & & $4.5 \pm 0.9$ & $19.0 \pm 1.6$ &
\end{tabular}

TABLE 8

OBSERVED BEHAVIOUR OF FEMALES ACCORDING TO ORDER

\begin{tabular}{lrrrrrr}
\hline \hline Order & Avg. error & $\begin{array}{c}\text { Max } \\
\text { beats }\end{array}$ & $\begin{array}{c}\text { Avg. } \# \text { of } \\
\text { beats }\end{array}$ & $\begin{array}{c}\text { Avg. } \# \\
\text { turns }\end{array}$ & \\
\hline 1 & $4.2 \pm 4.3$ & 36 & & $7.2 \pm 5.0$ & $19.0 \pm 5.7$ & \\
2 & $2.6 \pm 3.2$ & 31 & $5.1 \pm 4.2$ & $22.0 \pm 6.4$ & \\
3 & $2.5 \pm 2.5$ & 16 & $4.8 \pm 3.1$ & $21.7 \pm 5.1$ &
\end{tabular}

\section{Game type}

For male participants, the total number of beats decreased with the increasing amount of gestures in the games. These results suggest that, as the number of Kaspar's gestures increased, they tended to focus more interaction and less on than drumming (Table 9).

TABLE 9

OBSERVED BEHAVIOUR OF MALES ACCORDING TO GAME TYPE

\begin{tabular}{llrrrr}
\hline \hline Game type & $\begin{array}{l}\text { Avg. } \\
\text { error }\end{array}$ & $\begin{array}{l}\text { Max } \\
\text { beats }\end{array}$ & $\begin{array}{l}\text { of } \\
\text { beats }\end{array}$ & $\begin{array}{l}\text { Avg. \# of } \\
\text { Avg. \# of turns }\end{array}$ \\
\hline no-gesture & $2.5 \pm 2.7$ & 41 & & $7.3 \pm 5.8$ & $16.2 \pm 6.1$ \\
gesture & $3.9 \pm 3.6$ & 37 & & $6.3 \pm 4.8$ & $16.3 \pm 5.8$ \\
gesture + & $3.0 \pm 2.9$ & 9 & & $5.7 \pm 2.6$ & $15.8 \pm 4.2$
\end{tabular}

Different from the male participants, for females the total number of beats increased as the amount of gestures in the game increased. This indicates that the female participants tended to become more involved in the drumming with increases in non-verbal interaction gestural cues. The detailed evaluations are presented in Table 10.

TABLE 10

OBSERVED BEHAVIOUR OF FEMALES ACCORDING TO GAME TYPE

\begin{tabular}{|c|c|c|c|c|}
\hline Game type & $\begin{array}{l}\text { Avg. } \\
\text { error }\end{array}$ & $\begin{array}{l}\text { Max \# of } \\
\text { beats }\end{array}$ & $\begin{array}{l}\text { Avg. \# of } \\
\text { beats }\end{array}$ & Avg. \# of turns \\
\hline no-gesture & $2.7 \pm 2.5$ & 16 & $5.0 \pm 3.0$ & $21.0 \pm 4.8$ \\
\hline gesture & $3.1 \pm 3.8$ & 36 & $5.8 \pm 5.0$ & $21.3 \pm 4.7$ \\
\hline gesture+ & $3.5 \pm 3$ & 31 & $6.3 \pm 4.7$ & $20.3 \pm 7.8$ \\
\hline
\end{tabular}

It is interesting to note that, although the error rate in gesture+ was less than in the gesture condition, male participants liked it the least overall. They thought too many gestures distract them from drumming, instead of enjoying the 
gestures. Although gesture had the worst error rate, overall they liked it the most. In contrast, although the error rate in gesture+ was the highest, female participants liked it more than the no-gesture game which had the lowest error rate (Table 10).

\section{CONCLUSIONS}

In this work, we introduced a computational model of an imitative rhythmic interaction game using non-verbal gestural head, neck, and blinking gestures and deterministic turntaking between a robot and human partner. We based our model on drumming, which is a very suitable task for testing human-robot interaction. It is intended as more than a simple drumming synchronization task. In the long-term, we aim to develop social interaction between the robot and the human partner, which would not simply focus on synchronization to produce the same tempo, but result in producing a joyous and fruitful experience, while allowing us to gain insight into the role of non-verbal gesture in sustaining and regulating humanrobot interaction.

We used drumming interaction games enriched with different amounts of Kaspar's gestures to motivate the humans. In our experiments, we saw that humans are, in fact motivated by gestures and take enjoyment from this sharing. Too many gestures, however, break their concentration. Drumming with no gestures is considered successful by participants in terms of a drumming task but it is not considered successful in terms of social interaction. The results from this experiment thus highlight the possible tradeoff between the participants' subjective evaluation of the drumming experience, compared to objective measurements of the drumming performance, also reflecting individual preferences as to task and interactional aspects of the task. These results point towards a clear role for the use of appropriate amount and types of non-verbal gestures as a means of motivating drumming behaviour and regulating the interaction when interacting with a robot.

The reason for the high error rates at the start of the games is probably due to the human partner's high expectations from the game. Especially the male partners appeared to view this experiment not as a game, but rather a task to complete. Also, due to their background the human partners might have tried to 'test' the robot's limitations. So they initially played very fast, and very long sequences, and used different parts of the drum to enrich their play. They expected Kaspar to watch, understand and imitate them (most of the human partners thought the robot could detect them with its eye cameras and that the gestures were meaningful). As they played more, they understood the limited capabilities of the robot and modified their drumming and tried to synchronize with it.

Both the female and male participants overall liked the games with gestures, which had the worst error rates in the evaluations. This shows that the right amount of gestures would attract their attention, and make their experience enjoyable, although it did not actually help their drumming.
This reveals a strong difference between the subjective evaluations of the drumming experience by the participants, compared to objective performance measures.

This work is a first step in human-robot interaction research on synchronization, timing, and turn-taking using drumming games. Although we started with a simple implementation, the results are unexpected and interesting. As explained above, in our setup Kaspar just repeated the beats produced by the human partner, and made simple fixed head gestures accompanying its drumming (we especially used very simple gestures, not complex ones like smiling or frowning in order not to affect the human participants too much). The human partners' in return, perceived these simple behaviours as more complex and meaningful. They adapt themselves to the system unconsciously.

It is important to note that while Kaspar's drum playing did not change over time, and stayed the same in different games, the participants learned the limits of Kaspar and the rules of the game, and adapted themselves to the game better, so the success rate improved over time. Humans, as shown here, were not passive subjects in this game, but adapted themselves unconsciously to the capabilities of the robot. In order to facilitate and motivate such adaptation, aspects of the interaction that are not directly related to the task itself, such as interactional gestures may play an important role.

\section{FUTURE WORK}

Based on these results, future work on the humanoid drumming system will involve further study of the use of gestures for motivating the human partners. Because of our promising results from using gestures, we foresee a system wherein Kaspar may be motivated and rewarded by the human partner, through the partner's gestures and other expressive actions, and respond to these by playing novel acoustic rhythms and using its own repertoire of expressions and gestures to show satisfaction with these interactions. If our initial results can be extrapolated, then such a system will be even more capable of motivating and sustaining interaction.

\section{ACKNOWLEDGEMENTS}

This work was conducted within the EU Integrated Project RobotCub ("Robotic Open-architecture Technology for Cognition, Understanding, and Behaviours"), funded by the EC through the E5 Unit (Cognition) of FP6-IST under Contract FP6-004370. We would like to thank our colleagues in the Adaptive Systems research group for help in preparing the experiments and the paper: Michael L. Walters, Joe Saunders, N. Assif Mirza, Qiming Shen, Aris Alissandrakis, Nuno Otero and Ester Ferrari.

\section{REFERENCES}

[1] G. Weinberg, S. Driscoll and Parry, M. (2005). Musical interactions with a perceptual robotic percussionist. Proceedings of IEEE International Workshop on Robot and Human Interactive Communication (RO-MAN 2005) Nashville, TN, pp. 456-461. 
[2] G. Weinberg, and Driscoll, S. (2006). Robot-human interaction with an anthropomorphic percussionist. Proceedings of International ACM Computer Human Interaction Conference (CHI 2006). Montreal, Canada, pp.1229-1232.

[3] C. Crick, M. Munz, and Scassellati, B. (2006). Synchronization in social tasks: Robotic drumming. Proceedings of IEEE RO-MAN 2006, pp. 97102.

[4] S. Degallier, C. P. Santos, L. Righetti and A. Ijspeert. (2006). Movement generation using dynamical systems: a humanoid robot performing a drumming task. In Proceedings of the IEEE-RAS International Conference on Humanoid Robots (HUMANOIDSO6).

[5] S. Kotosaka, and Schaal, S. (2000). Synchronized robot drumming by neural oscillator. International Symposium on Adaptive Motion of Animals and Machines.

[6] T. W. Bickmore \& Cassell, J. (2005). Social Dialogue with Embodied Conversational Agents. In: J. v. Kuppevelt, L. Dybkjaer \& N. Bernsen (Eds.), Natural, Intelligent and Effective Interaction with Multimodal Dialogue Systems. New York: Kluwer Academic, pp. 23-54.

[7] T. W. Bickmore \& Picard, R. W. 2005. Establishing and Maintaining Long-Term Human Computer Relationships. ACM Transactions on Computer-Human Interaction 12(2): 293-327.

[8] A. Tapus \& Mataric', M. J. (2006). Towards Socially Assistive Robotics. International Journal of the Robotics Society of Japan 24(5), pp.576578

[9] C. Breazeal (2002). Designing Sociable Robots. MIT Press.

[10] M.P. Michalowski, S. Sabanovic and Michel, P. (2006). Roillo: Creating a social robot for playrooms. Proceedings of IEEE RO-MAN 2006, pp.587-592.

[11] H. Kozima, C. Nakagawa, Y. Yasuda, and Kosugi, D. (2004). A toy-like robot in the playroom for children with developmental disorder. Proceedings of the International IEEE Conference on Development and Learning (ICDL-04, San Diego, USA).

[12] KEEPON. (2007) http://univ.nict.go.jp/people/xkozima /infanoid/robot-eng.html\#keepon.

[13] B. Robins, K. Dautenhahn, C. L. Nehaniv, N. A. Mirza, D. Francois, and Olsson, L. (2005). Sustaining interaction dynamics and engagement in dyadic child-robot interaction kinesics: Lessons learnt from an exploratory study. In Proc. of the 14th IEEE International Workshop on Robot and Human Interactive Communication, ROMAN2005, pp. 716722.

[14] M.P. Blow, K. Dautenhahn, A. Appleby, C. Nehaniv, D. Lee (2006). Perception of robot smiles and dimensions for human-robot interaction design. Proc. 15th IEEE International Symposium on Robot and Human Interactive Communication (RO-MANO6), pp.469-474.

[15] G. Metta, P. Fitzpatrick, and Natale, L. (2006). Yarp: yet another robot platform. International Journal on Advanced Robotics Systems, Special Issue on Software Development and Integration in Robotics, 3(1), pp. 43-48.

[16] Portaudio (2007). http://www.portaudio.com/trac/wiki/.

[17] H. Kose, and Akin, H. L. (2001). Object recognition in robot football using a one-dimensional image. The Tenth Turkish Symposium on Artificial Intelligence and Neural Networks (TAINN 2001), pp.291-300. 